\title{
Evidence of a cholinergic input into the suppression of the transport response in white rats
}

\author{
CHRISTOPHER WILSON and ALLISON D. CROMEY \\ Lafayette College, Easton, Pennsylvania
}

\begin{abstract}
A series of five experiments were performed to investigate the hypothesis that the maturation of forebrain cholinergic systems results in the normal suppression of "transport response" intensity reported in rat pups at about Days 28-30. In the first experiment, we administered the cholinergic antagonist atropine sulfate to 30 -day-old rats, and recorded a subsequent increase in transport response intensity as compared with that of saline-treated control rats. In the second experiment, we administered varying doses of the cholinergic agonist pilocarpine nitrate to 23day-old rats, and recorded a subsequent suppression of the transport response with both low and high doses of the drug. In the third experiment, we administered atropine to 23-day-old rat pups, and recorded no changes in the transport response, which indicates that the results of Experiment 1 were not due to a side effect of the drug administered. In the fourth experiment, we administered pilocarpine to 30-day-old rats and recorded decreases in transport response intensity, which indicates that this behavioral activity is related to the maturation of the cholinergic system. In the last experiment, we administered pilocarpine in combination with either the peripheral cholinergic blocker methylscopolamine hydrobromide or the central cholinergic blocker scopolamine hydrochloride. We found that scopolamine hydrochloride blocked the decrease in transport response intensity seen with pilocarpine, but that methylscopolamine hydrobromide had no effect on the reduction produced by pilocarpine. These results indicate that the nature of the transport response is one of behavioral arousal, and that the rapid reduction in response intensity, beginning at the time of weaning, is dependent upon the maturation of forebrain cholinergic systems.
\end{abstract}

In developing altricial animals, the various neurotransmitter systems become mature at different times, with the typical pattern of functional maturity following a caudal-to-rostral sequence (Campbell, Lytle, \& Fibiger, 1969; Kellogg \& Lundborg, 1972; Loizou, 1969, 1972; Moorcroft, Lytle, \& Campbell, 1971). For instance, dopamine and norepinephrine cell bodies associated with the midbrain reticular system are extant at or near birth (Campbell et al., 1969; Coyle \& Axelrod, 1972; Siegel, Albers, Agranoff, \& Katzman, 1981), whereas forebrain serotonergic and cholinergic systems are not functional until several weeks postpartum (Campbell et al., 1969).

In examining age-related behavioral changes, one must consider the interaction of the various neurotransmitter systems in producing and/or modulating particular behaviors or classes of behavior (Carlton, 1963). In the developing rat, for instance, as the dopamine systems mature, functionally related increases in behavioral arousal appear (Campbell et al., 1969; Kellogg \& Lundborg, 1972; Lal \& Sourkes, 1973) along with various forms of stereotypy, including sniffing, licking, gnawing, and wall-climbing (Barrett, Caza, Spear, \& Spear, 1982; Costall \& Naylor, 1973; Ernst, 1967; Lal \&

Requests for reprints should be addressed to Christopher Wilson, Department of Psychology, Lafayette College, Easton, PA 18042.
Sourkes, 1973; Melzacka, Wisniowska, \& Vetulani, 1978; Reinstein, McClearn, \& Isaacson, 1978). As the animal continues to mature, there is a decrease in dopamine-related behavioral activation, which appears to be a modulating function imposed by the development of forebrain cholinergic systems (Blozovski \& Bachevalier, 1975; Carlton, 1963; Fibiger, Lytle, \& Campbell, 1970; Moorcroft et al., 1971). Thus, cholinergic systems appear to have a modulating effect on dopaminergic systems with respect to behavioral arousal (Campbell \& Mabry, 1973; Fibiger et al., 1970).

Brewster and Leon (1980) described a stereotypic behavioral response in rat pups that helps the mother transport the pups from one location to another. This behavior is characterized by an active flexion and adduction of the hindlimbs, and an extension and adduction of the forelimbs, and is made in response to being orally grasped by the nape of the neck. This "transport response" is elicited maximally by dorsal stimulation initiated by the mother, and is beneficial because it occurs when the pups are fairly active and relatively unwieldy (Brewster \& Leon, 1980; Wilson, 1985; Wilson, Cullen, \& Sendell, 1984). Thus, the response is ecologically important.

The development of the transport response falls within the time frame typically associated with that of behavioral activation and the development of the dopamine system. The response first becomes evident at about Postnatal 
Day 8 , gradually increasing in frequency and intensity until about Day 20, at which time it begins to wane (Brewster \& Leon, 1980). The response also appears to be subserved by a dopamine system, because it can be abolished with haloperidol in 19-day-old rat pups (Wilson et al., 1984) and reinstated with the administration of apomorphine in 40-day-old rats, animals typically too old to show the transport response (Wilson, 1985). Interestingly, a weak transport response can be potentiated with stimuli that normally elicit behavioral activation (Wilson, 1988).

The transport response gradually decreases in intensity and is difficult to elicit by the end of the 4th postpartum week (Brewster \& Leon, 1980), a time when forebrain cholinergic systems reach functional maturity (Fibiger et al., 1970). The series of experiments presented here were designed to determine if the reduction in frequency and intensity of the transport response in rats, which occurs at about Day 30, is a function of the maturation of cholinergic systems. Experiment 1 was designed to investigate the effect of blocking the cholinergic system on the ease of eliciting the transport response in 30-day-old rats, animals normally too old to show a strong response. Experiment 2 was designed to investigate the effect of stimulating the central cholinergic system on the ease of eliciting the transport response in 23-day-old rats, animals that would normally show a fairly intense response. Experiments 3 and 4 were designed to provide data to support the conclusions derived from the results of the previous experiments, and to test the possibility that the effects reported in the previous experiments were not due to side effects of the drugs administered. Experiment 5 was designed to test the hypothesis that the effects of pilocarpine reported in Experiment 2 were in fact a function of central cholinergic system stimulation.

\section{METHOD}

\section{Subjects}

The subjects in these experiments consisted of 176 SpragueDawley albino rats, either 23 or 30 days of age at the time of testing. Litters were derived from breeding colonies in the Department of Psychology at Lafayette College. The litters were housed with their mothers in clear Plexiglas breeding cages in a room kept at $22{ }^{\circ} \mathrm{C}$, and were kept on a 12:12 h light:dark cycle with lights on at $0700 \mathrm{~h}$. All testing occurred between 1000 and $1200 \mathrm{~h}$, with each rat being tested at only one age and one drug dosage.

\section{Materials}

Atropine sulfate, scopolamine hydrochloride, and pilocarpine nitrate were purchased from Sigma Chemical Co. Methylscopolamine hydrobromide was generously donated by The Upjohn Co.

\footnotetext{
General Procedure

Approximately 4 days prior to parturition, pregnant female rats were placed in clear Plexiglas breeding chambers that contained nesting material. Each day, at 0900 and $1700 \mathrm{~h}$, the cages were checked for the presence of newborn litters. The first day a litter was present was recorded as Postnatal Day 0. On the morning of Postnatal Day 1, each litter was culled to 8-10 pups; litters that contained fewer than 8 pups were not used in these experiments.
}

On the testing day, the dam was removed from the litter, and the pups were transferred to an alternate experimental room and placed in a breeding cage with fresh nesting material. The pups were then given the appropriate drug treatments as outlined in the experiments below.

Following the respective drug administration regimen, each rat was given four postdrug trials, with approximately 2-min intertrial intervals, to test for the occurrence and intensity of the transport response. In this test, an experimenter grasped the skin at the nape of the neck of the pup between his/her thumb and first two fingers and then lifted and suspended the pup in that position. The intensity of the pup's transport response was graded on a scale of 0-5, according to the schema proposed by Brewster and Leon (1980). This schema was designed to award 1 point for each forelimb, hindlimb, and/or tail that the animal brought into contact with its ventral surface. The mean of the four trials was used for statistical analyses.

\section{Data Analysis}

The data were analyzed using standard parametric procedures, ${ }^{1}$ with subsequent comparisons between conditions being determined with Newman-Keuls tests (Kirk, 1968). Differences with a probability of less than $.05(p<.05)$ were considered statistically reliable.

\section{EXPERIMENT 1}

Carlton (1963) demonstrated that scopolamine could potentiate the arousal effects of subthreshold doses of amphetamines in rats. Fibiger et al. (1970) reported that scopolamine potentiated the arousal effects of amphetamines, but that this effect only occurred in animals 25 days of age or older. Likewise, Blozovski and Bachevalier (1975) reported that atropine produced a significant potentiation of dopamine-related stereotypic behaviors in animals 25 days old or older, with a maximal action of the drug occurring at postnatal day 28 . The present experiment was designed to determine if reductions in the dopamine-related transport response, which occur at about Days 25-30, could be prevented by blocking endogenous cholingergic systems. Specifically, in this experiment, 30day-old rats were given varying doses of atropine sulfate and were then tested for the presence and intensity of the transport response. We hypothesized that if the reduction in the transport response was due to the maturation of endogenous cholinergic systems, rats receiving higher doses of atropine should show an increase in transport response intensity.

\section{Procedure}

Forty-eight 30-day-old rat pups were tested using a factorial design. The pups were separated from their mothers and placed in a litter cage containing fresh litter. They were then given intraperitoneal (i.p.) injections of varying doses $(0.0,5.0,10.0$, or $15.0 \mathrm{mg} / \mathrm{kg} / 5 \mathrm{ml}$ ) of atropine sulfate. After a 20 -min postinjection delay, the pups were tested for the presence and intensity of the transport response as described above.

\section{Results and Discussion}

Mean response intensities for the subjects in this experiment are presented in Table 1. It appears that there was a dose-dependent increase in transport response intensity with increasing doses of the cholinergic antagonist 
Table 1

Mean Response Intensities and Standard Errors as a Function of Dosage of Atropine Sulfate in 30-Day-Old Rat Pups

\begin{tabular}{ccc}
\hline $\begin{array}{c}\text { Dose of } \\
\text { Atropine Sulfate } \\
(\mathrm{mg} / \mathrm{kg} / 5 \mathrm{ml})\end{array}$ & \multicolumn{2}{c}{ TR Intensity } \\
\hline 0.0 & $M$ & $S E$ \\
5.0 & 0.85 & .27 \\
10.0 & 1.52 & .29 \\
15.0 & $1.88^{*}$ & .20 \\
\hline
\end{tabular}

* Reliably different from control condition.

atropine. An analysis of variance (ANOVA) analyzing the differences in mean response intensities yielded a significant dose effect $[F(3,44)=6.76, p<.05]$. NewmanKeuls post hoc analyses revealed that, relative to the saline-control rats, rats that had received 10 and $15 \mathrm{mg} / \mathrm{kg}$ of atropine sulfate showed a significant increase in response intensity. No other differences were statistically reliable. These data support the hypothesis that the reduction in transport response intensity occurring at about Days $28-30$ is the result of the maturation of cholinergic systems.

\section{EXPERIMENT 2}

Hedley and Wallach (1983) reported an attenuation of apomorphine-induced stereotypic behaviors in mice that were given pretreatments of the cholinomimetic pilocarpine and the anticholinesterase agent physostigmine. Likewise, Fibiger et al. (1970) reported an attentuation of amphetamine-induced psychomotor activity in 20-25-dayold rats following the administration of pilocarpine. The present experiment was designed to test the hypothesis that maturation of the cholinergic system results in a suppression of the transport response. Specifically, in this experiment 23-day-old rats were given varying doses of pilocarpine and were then tested for the presence and intensity of the transport response. We chose 23-day-old rats to test the effects of pilocarpine because we wanted to induce a system that was capable of being stimulated but was not completely functional endogenously, at the time of testing. We hypothesized that if the reduction in the transport response is due to the maturation of forebrain cholinergic systems, then the administration of pilocarpine would result in a decrease in the transport response intensity in rats.

\section{Procedure}

Forty 23-day-old rat pups were tested using a factorial design. The pups were separated from their mothers and placed in a litter cage containing fresh litter, and were then given i.p. injections of varying doses $(0.0,1.0,2.5$, or $5.0 \mathrm{mg} / \mathrm{kg} / 5 \mathrm{ml})$ of pilocarpine nitrate. Following a $20-\mathrm{min}$ postinjection delay, the pups were tested for the presence and intensity of the transport response as described above.

\section{Results and Discussion}

Mean response intensities of subjects in this experiment are presented in Table 2 . The data appear to show a reduc- tion in transport response intensity following the administration of the cholinomimetic pilocarpine. An ANOVA analyzing the differences in mean response intensities yielded a significant dose effect $[F(3,36)=5.93$, $p<.05]$. Post hoc analyses revealed that, relative to the saline-control rats, rats that had received 1.0 and $5.0 \mathrm{mg} / \mathrm{kg}$ of pilocarpine nitrate showed a significant reduction in response intensity. These data are similar to those reported by Fibiger et al. (1970).

The lack of a significant effect in response intensity at the $2.5 \mathrm{mg} / \mathrm{kg}$ pilocarpine dose is troubling. We reexamined the individual trials and found that 3 of the animals in the $2.5-\mathrm{mg} / \mathrm{kg}$ group emitted fairly intense responses to the pinch; the other rats in this group consistently showed weak-to-moderate response intensities. This deviation is evident in the fairly large standard error for that group, the largest standard error for any of the groups reported in this study. We are not sure why such a large percentage of the $2.5-\mathrm{mg} / \mathrm{kg}$ subjects emitted strong responses, and we are continuing to look at this dosage to determine if there was some error variance in our drug/stimulus administration or in our scoring procedures. Across groups for this experiment, the data reported here indicate that we can induce a premature suppression of the transport response by stimulating the cholinergic system, and that the response suppression seen 5-7 days later may be a function of normal endogenous cholinergic maturation.

\section{EXPERIMENT 3}

Experiments 1 and 2 suggest a cholinergic involvement in the normal suppression of the transport response. In Experiment 2, we asserted that the transport response was very strong in 23-day-old rats because forebrain cholinergic systems had not yet matured. We reasoned that pilocarpine administration to these animals suppressed the transport response because of the presence of forebrain cholinergic receptors (Fibiger et al., 1970). If these assertions are correct, then the administration of atropine sulfate should have little effect on the transport response in 23-day-old rat pups. Experiment 3 was designed to test this assertion. Specifically, in this experiment, 23-dayold rat pups were given either a very high dose of atropine sulfate or a saline vehicle solution. In Experiment 1, we reported a dose-dependent increase in the transport

Table 2

Mean Response Intensities and Standard Errors as a Function of Dosage of Pilocarpine Nitrate in 23-Day-Old Rat Pups

\begin{tabular}{ccc}
\hline $\begin{array}{c}\text { Dose of } \\
\text { Pilocarpine Nitrate } \\
(\mathrm{mg} / \mathrm{kg} / 5 \mathrm{ml})\end{array}$ & \multicolumn{2}{c}{ TR Intensity } \\
\cline { 2 - 3 } 0.0 & $M$ & $S E$ \\
1.0 & 2.63 & .23 \\
2.5 & $1.25^{*}$ & .30 \\
5.0 & 2.20 & .42 \\
& $1.05^{*}$ & .26 \\
\hline
\end{tabular}

* Reliably different from control condition. 
response following the administration of atropine sulfate in 30-day-old animals. In the present experiment, we chose to compare transport response intensities in pups that had received a saline vehicle administration with those in pups that had received the dose of atropine sulfate that produced the maximal effect in Experiment $1(15 \mathrm{mg} / \mathrm{kg})$. We hypothesized that if the intense transport response in 23-day-old pups was due, in part, to the relative immaturity of the cholinergic system, the administration of atropine sulfate would have no effect on these animals. If there is an increase in transport response intensity after the administration of atropine sulfate, then an alternate explanation may be in order.

\section{Procedure}

Twenty 23-day-old rat pups were tested using a factorial design. The pups were separated from their mothers and placed in a cage containing fresh litter, and were then given i.p. injections of either 0.0 or $15.0 \mathrm{mg} / \mathrm{kg} / 5 \mathrm{ml}$ of atropine sulfate. Following a $20-\mathrm{min}$ postinjection delay, the pups were tested for the presence and intensity of the transport response as described above.

\section{Results and Discussion}

Mean transport response intensities for the subjects in this experiment are presented in Table 3. An independent $t$ test revealed a nonreliable effect of the atropine on the transport response in these rats $[t(18)=.15, p>.05]$. These data support the assertion that the reduction in transport response intensity occurring at about Days 28-30 was a result of cholinergic maturation, and not a side effect of the cholinergic blocker atropine. This result was expected because our intention was to block a system that was not yet functionally extant.

\section{EXPERIMENT 4}

If the transport response is suppressed in 30-day-old rats because of the functional maturation of forebrain cholinergic systems, one would expect that the administration of pilocarpine to these animals would have little effect on the intensity of the response. The present experiment was designed to test this assertion by comparing transport response intensities in 30-day-old subjects that had been given $5.0 \mathrm{mg} / \mathrm{kg}$ of either saline or pilocarpine nitrate. We decided to use $5.0 \mathrm{mg} / \mathrm{kg}$ of pilocarpine because that was the dose that had given the maximal effect in Experiment 2.

\section{Procedure}

Twenty 30-day-old rats were tested using a factorial design. The rats were separated from their mothers and placed in breeding cages

Table 3

Mean Response Intensities and Standard Errors as a Function of Dosage of Atropine Sulfate in 23-Day-Old Rat Pups

\begin{tabular}{ccc}
\hline $\begin{array}{c}\text { Dose of } \\
\text { Atropine Sulfate } \\
(\mathrm{mg} / \mathrm{kg} / 5 \mathrm{ml})\end{array}$ & \multicolumn{2}{c}{ TR Intensity } \\
\cline { 2 - 3 } & $M$ & $S E$ \\
\hline 0.0 & 2.76 & .23 \\
15.0 & 2.80 & .10 \\
\hline
\end{tabular}

Table 4

Mean Response Intensities and Standard Errors as a Function of Dosage of Pilocarpine Nitrate in 30-Day-Old Rat Pups Dose of

Pilocarpine Nitrate $(\mathrm{mg} / \mathrm{kg} / 5 \mathrm{ml})$

0.0

5.0 TR Intensity

\begin{tabular}{cc}
$M$ & $S E$ \\
\hline 0.98 & .30 \\
0.53 & .16 \\
\hline
\end{tabular}

with fresh litter, and were then given i.p. injections of $5.0 \mathrm{mg} / \mathrm{kg} / 5 \mathrm{ml}$ of either saline or pilocarpine nitrate. Following a 20-min postinjection delay, the pups were tested for the presence and intensity of the transport response as described above.

\section{Results and Discussion}

Mean response intensities for the subjects in this experiment are presented in Table 4. The data appear to show a moderate reduction in the transport response with the administration of pilocarpine in these 30-day-old animals. A $t$ test for independent groups revealed a nonreliable reduction in the transport response for subjects in the pilocarpine-administered group $[t(18)=1.32$, $p>$.05]. This reduction was not completely expected, inasmuch as our manipulation was intended to stimulate a system that was already functioning effectively. The subjects in the saline-control group showed relatively low response intensities, whereas the subjects in the pilocarpine group showed virtually no response to the tactile stimulation. Our assumption is that the cholinergic and dopaminergic systems of 30-day-old rats are in competition with respect to the transport response. These animals will generally show a very weak transport response lending support to the assertion that some dopaminergic activity, albeit at a very low level, produces the response. Administration of pilocarpine to these animals may cause the cholinergic system to have a much greater influence on the animals' behavioral repertoire, and result in a virtually complete suppression of the response. Brewster and Leon (1980) reported that the transport response could be prolonged by particular manipulations, such as prolonged handling. These manipulations may cause the dopaminergic system to have a greater influence on the animals' behavioral repertoire, thus increasing the probability that the transport response will be exhibited.

\section{EXPERIMENT 5}

Data from the four previous experiments point to the conclusion that the maturation of forebrain cholinergic systems underlies the transport response suppression found in 25-30-day-old rats. The fact that we found no reduction in the transport response in subjects in Experiment 3 indicates that the effects obtained with the administration of atropine are not due to peripheral cholinergic blocking. Experiment 5 was designed to test the hypothesis that the effects reported here were dependent upon the manipulation of a central cholinergic system, and were not due to incidental manipulation of a peripheral system. In Experiment 5, the transport response intensity of 23- 
day-old rats that were administered pilocarpine nitrate after having been injected with either a peripheral cholinergic blocker-methylscopolamine hydrobromide-or a central cholinergic blocker-scopolamine hydrochloride-was compared with that of control subjects. We hypothesized that, if the effects reported in the previous experiments were dependent upon the manipulation of central cholinergic mechanisms, then the response of animals that had received methylscopolamine hydrobromide with pilocarpine would be similar to that of animals that had received pilocarpine nitrate alone. We further hypothesized that the response of animals that had received scopolamine hydrochloride with pilocarpine would be similar to that of animls that received saline alone.

\section{Procedure}

Forty-eight 23-day-old rats were divided into six groups using a factorial design. The subjects in this experiment were given a series of two injections, the first an i.p. administration of saline, methylscopolamine hydrobromide $(1.0 \mathrm{mg} / \mathrm{kg} / 5 \mathrm{ml})$, or scopolamine hydrochloride $(1.0 \mathrm{mg} / \mathrm{kg} / 5 \mathrm{ml})$, and the second, 5 min later, an i.p. administration of either saline or pilocarpine nitrate $(5.0 \mathrm{mg} / \mathrm{kg} / 5 \mathrm{ml})$. The six groups thus consisted of rats that received: (1) saline followed by saline; (2) saline followed by pilocarpine nitrate; (3) methylscopolamine hydrobromide followed by saline; (4) methylscopolamine hydrobromide followed by pilocarpine nitrate; (5) scopolamine hydrochloride followed by saline; (6) scopolamine hydrochloride followed by pilocarpine nitrate. Following a 20-min postinjection delay, the subjects were tested for the presence and intensity of the transport response as described above.

\section{Results and Discussion}

The results of this experiment are presented in Table 5. The data appear to show a reduction in transport response intensities for subjects that received pilocarpine, regardless of whether they had received prior administrations of methylscopolamine hydrobromide or saline. However, this reduction in transport response intensity appears to be blocked by the prior administration of scopolamine hydrochloride. An ANOVA confirmed these observations, yielding a reliable group effect $[F(5,42)=16.57$, $p<.05]$. Post hoc analyses revealed significant reductions in response intensities for the subjects in the methylscopolamine/pilocarpine and saline/pilocarpine groups, as opposed to response intensities for the subjects in all other groups. No other differences were statistically reliable.

Table 5

Mean Response Intensities and Standard Errors as a Function of Drug Condition in 23-Day-Old Rat Pups

\begin{tabular}{lcc}
\hline \multirow{2}{*}{\multicolumn{1}{c}{ Drug Condition }} & \multicolumn{2}{c}{ TR Intensity } \\
\cline { 2 - 3 } & \multicolumn{1}{c}{$M$} & $S E$ \\
\hline Saline/Saline & 2.66 & .32 \\
Saline/Pilocarpine & $0.91^{*}$ & .29 \\
Methylscopolamine OHBr/Saline & 3.03 & .34 \\
Methylscopolamine OHBr/Pilocarpine & $0.97^{*}$ & .25 \\
Scopolamine HCl/Saline & 3.28 & .12 \\
Scopolamine HCl/Pilocarpine & 2.91 & .19
\end{tabular}

* Reliably different from saline/saline condition.
These data indicate that the reduction in transport response intensity produced by pilocarpine in 23-day-old rats is the result of the stimulation of central cholinergic systems. This conclusion is supported by the fact that blocking the peripheral effects of the drug did not reverse the effect of pilocarpine, whereas blocking the central effects of the drug did block the effect of pilocarpine.

\section{GENERAL DISCUSSION}

The transport response in infant rats begins at about Day 8 , increases in intensity until about Day 20 or Day 21, and then decreases until about Day 30, after which it can no longer be elicited (Brewster $\&$ Leon, 1980). These changes in transport response intensity follow the same general progression as that of spontaneous locomotor activity, as reported by Moorcroft et al . (1971). In addition, specific stereotypic responses to external stimuli normally associated with behavioral arousal in rats aged 9 days and older have been reported. Hall (1979) reported a variety of behaviors, including probing, licking, and mouthing following milk infusions into the oral cavity in 9-20-day-old rat pups. Antelman and Szechtman (1975) reported the induction of eating, gnawing, and licking that was elicited in adult rats with a mild tailpinch. Recently, we have reported that a tailpinch can potentiate an initially weak transport response in 15-dayold rats (Wilson, 1988).

The increases in behavioral arousal seen early in development appear to be dopaminergically mediated (Antelman \& Szechtman, 1975; Camp \& Rudy, 1987), and subsequent decreases appear to result from forebrain cholinergic modulation (Fibiger et al., 1970). We have reported that dopaminergic activity appears to underlie the emergence of the transport response in young rats (Wilson, 1985; Wilson et al., 1984). In the present study, we now report progressive increases in transport response intensity with the administration of increasing doses of the cholinergic-receptor blocker atropine in 30-day-old rats. However, if atropine is given prior to the time that forebrain cholinergic systems become functionally mature, at 23 days of age, it has no effect on transport response intensity. These results lead us to the conclusion that the progressive decrease in transport response intensity that occurs at around Days 28-30 is a function of forebrain cholinergic maturation.

Fibiger et al. (1970) reported that the administration of pilocarpine to 20 - and 25 -day-old rats produced a reduction in amphetamine-induced behavioral arousal as measured by stabilimeter activity. This finding led Fibiger et al. to the conclusion that telencephalic cholinergic inhibitory systems become functional between Days 20 and 25. The results reported in Experiment 2 of the present study are similar to the data on behavioral arousal for the 20-day-old rats that were presented by Fibiger et al.; there was a marked reduction in response intensity at two of the three doses of pilocarpine in our study. These data, along with the results of Experiment 4, support the con- 
clusion that the suppression of the transport response that normally occurs during the 4 th and 5 th postpartum weeks is cholinergically mediated.

Finally, the results from Experiment 5 demonstrate that pilocarpine, when administered in conjunction with the peripheral cholinergic blocker methylscopolamine hydrobromide, continues to produce consistent reductions in the transport response in 23-day-old rats. Moreover, the central cholinergic blocker scopolamine hydrochloride blocked the reduction in transport response intensity, when administered with pilocarpine nitrate in 23-day-old animals. Our results provide further evidence that the effects reported in the aforementioned studies are the results of the actions of the specific drugs on the central nervous system.

Meyer, Smith, and Van Hartesveldt (1984) suggested that, in rats, the transport response may be related to the dorsal immobility response. In investigating the mechanisms that underlie the transport response, we have found that with a pinch to the nape of the neck, the animals become very quiet, but that the limb response is one of active flexion/extension, with the rats maintaining the limb adduction posture. We have reported that stimuli normally associated with behavioral arousal can potentiate an initially weak transport response (Wilson, 1988). We have also implicated a central dopaminergic mechanism in the initiation of the transport response (Wilson, 1985). With this study, we implicate a central cholinergic mechanism in the suppression and eventual disappearance of the transport response. Thus, we have reported both ecological and pharmacological evidence that links the transport response to behavioral arousal. We are currently investigating some possible relationships of the transport response to the dorsal immobility response. We are also investigating other conditions under which the response can be intensified or weakened, and the underlying neurochemical mechanisms to help us better characterize and categorize this ecologically significant behavior.

\section{REFERENCES}

Antelman, S. M., \& Szechtman, H. (1975). Tail pinch induces eating in sated rats which appears to depend on nigrostriatal dopamine. Science, 189, 731-733.

Barrett, B. A., Caza, P., Spear, N. E., \& Spear, L. P. (1982). Wall climbing, odors for the home nest, and catecholaminergic activity in rat pups. Physiology \& Behavior, 29, 501-507.

Blozovski, D., \& BACHEV ALIER, J. (1975). Effects of atropine on behavioral arousal in the developing rat. Developmental Psychobiology, 8, 97-102.

Brewster, J., \& Leon, M. (1980). Facilitation of maternal transport by Norway rat pups. Journal of Comparative \& Physiological Psychology, 94, 80-88.

CAMP, L. L., RUDY, J. W. (1987). Behavioral activation in infant rats: Pharmacological evidence for dopaminergic mediation. Psychobiology, 15, 317-328.

Campbell, B. A., Lytle, L. D., \& Fibiger, H. C. (1969). Ontogeny of adrenergic arousal and cholinergic inhibitory mechanisms in the rat. Science, 166, 635-637.

Campbell, B. A., \& Mabry, P. D. (1973). The role of catecholamines in behavioral arousal during ontogenesis. Psychopharmacologia, 31, 253-264
Carlton, P. L. (1963). Cholinergic mechanisms in the control of behavior by the brain. Psychological Review, 70, 19-39.

Costall, B., \& Naylor, R. J. (1973). The role of telencephalic dopaminergic systems in the mediation of apomorphine-stereotyped behaviour. European Journal of Pharmacology, 24, 8-24.

Coyle, J. T., \& AXELrod, J. (1972). Dopamine-hydroxylase in the rat brain: Developmental characteristics. Journal of Neurochemistry, 19 , 449-459.

ERNST, A. M. (1967). Mode of action of apomorphine and dexamphetamine on gnawing compulsion in rats. Psychopharmacologia, 10, 316-323.

Fibiger, H. C., Lytle, L. D., \& CAmpbell, B. A. (1970). Cholinergic modulation of adrenergic arousal in the developing rat. Journal of Comparative \& Physiological Psychology, 72, 384-389.

HALL, W. G. (1979). The ontogeny of feeding in rats: I. ingestive and behavioral responses to oral infusions. Journal of Comparative \& Physiological Psychology, 93, 977-1000.

Hedley, L. R., \& Wallach, M. B. (1983). Potentiation of apomorphine-induced gnawing in mice. Progress in NeuroPsychopharmacology \& Biological Psychiatry, 7, 47-56.

Kellogg, C., Lundero, P. (1972). Ontogenetic variations in responses to 1-dopa and monoamine receptor-stimulating agents. Psychopharmacologia, 23, 187-200.

KIRK, R. E. (1968). Experimental design: Procedures for the behavioral sciences. Belmont, CA: Brooks/Cole.

LAL, S., \& SourKes, T. L. (1973). Ontogeny of stereotyped behavior induced by apomorphine and amphetamine in the rat. Archives of International Pharmacodynamics \& Therapeutics, 202, 171-182.

LoIzoU, L. (1969). The development of monoamine-containing neurones in the brain of the albino rat. Journal of Anatomy, London, 104, 588.

LoIzou, L. (1972). The postnatal ontogeny of monoamine-containing neurons in the central nervous system of the albino rat. Brain Research, 40, 395-418.

Melzacka, M., Wisniowska, G., \& Vetulani, J. (1978). The distribution of apomorphine in rat brain: Possible behavioral correlates. Polish Journal of Pharmacology, 30, 335-345.

Meyer, M. E., Smith, R. L., \& Van Hartesveldt, C. (1984). Haloperidol differentially potentiates tonic immobility, the dorsal immobility response, and catalepsy in the developing rat. Developmental Psychobiology, 17, 383-389.

Moorcroft, W. H., Lytle, L. D., \& Campbell, B. A. (1971). Ontogeny of starvation-induced behavioral arousal in the rat. Journal of Comparative \& Physiological Psychology, 75, 59-67.

Reinstein, D. K., McClearn, D., \& IsaAcson, R. L. (1978). The development of responsiveness to dopamine agonists. Brain Research, 150, 216-223.

Siegel, G. J., Albers, R. W., Agranoff, B. W., Katzman, R. (1981). Basic neurochemistry (3rd ed.). Boston: Little, Brown.

Wilson, C., Cullen, E., \& Sendell, K. (1984). A pharmacologic investigation of the "transport response" in the white rat. International Journal of Developmental Neuroscience, 2, 323-329.

WILSON, C. (1988). The effects of sensory stimulation in inducing or intensifying the "transport response" in white rats. Animal Learning \& Behavior, 16, 83-88.

Wilson, C., Cullen, E., \& Sendell, K. (1984). A pharmacologic investigation of the "transport response" in the white rat. Intemational Journal of Developmental Neuroscience, 2, 323-329.

\section{NOTES}

1. Appropriate non-parametric statistical techniques were applied to the data from each experiment as a check for the parametric statistical tests reported in the Results sections. The non-parametric test results were not reported, as they were identical to the parametric statistical test results.

(Manuscript received March 17, 1988; revision accepted for publication September 2, 1988.) 